of paerperal infection due to retention of lochia, unrelieved by carettage and intra-uterine douching, cured by aterine drainage. I may add that I had during the course of the illness the advantage of consultation with the late Professor Budin who took a very grave view and did not hesitate to describe it as la grande infeotion, that is to say, generalised puerperal infection. The accouchement-of a second childtook place simply and normally on June 18th at what may be termed the customary hour of $3 \mathrm{~A} . \mathrm{M}$. Following my usual practice I had entirely avoided vaginal examination from the onset of labour, the progress of which $I$ had followed by abdominal palpation. For three days the patient's condition was excellent. (Looking back it seems probable, however, that at this stage an abnormal diminution of the lochia might have been noticed or at least suspected.) On the fourth day there was a rise of temperature to $102^{\circ} \mathrm{F}$. with pain low down in the abdomen on the right side. As the bowels had not moved since the confinement an enema was given with good result and for 36 hours the temperature remained normal. On the evening of the sixth day there was a rise to $101^{\circ}$ and on the next day, during my absence, my friend, Dr. C. K. Au tin, saw the patient, the temperature being $103^{\circ} 6^{\circ}$ and the pulse 110 . He gave an intra-uterine douche by which a large amount-about half a litre-of brown turbid fluid was washed out. This in no way relieved the patient, at least in so far as her comfort was concerned, as it was immediately followed by a violent rigor, the temperature remaining: high and the pulse rapid. On the next morning I repeated the intra-uterine douche, similariy washing out much turbid fluid. Within half an hour the temperature rose from $100 \cdot 6^{\circ}$ to $104 \cdot 6^{\circ}$, the pulse to 128 , and an alarming and prolonged rigor occurred. Professor Budin saw the patient at 2 P.M., soon after a third intra-uterine douche had been given, and during his visit the third rigor took place. Professor Budin's prognosis was of the gloomiest, and he expressed the opinion that unless the uterus were at once curetted death would probably take place. At 6 P.M. the patient was anæesthetised and the uterus thoroughly cleaned out with the écouvillon or quillmop-the instrument preferred to the steel curette by both Budin and Doleris for this purpose. ${ }^{1}$ The curette brought no placental débris away and there was no evidence of sloughing or desp infection of the uterine mucous membrane. The cavity of the uterus was packed with iodoform gauze. That night the situation seemed saved, the condition of the patient was excellent, the temperature normal, and the pulse 84 . On the following morning, by Professor Budin's directions and by his assistant, the gauze was removed and an intra-uterine douche was given ( 40 grammes of tincture of iodine and 6 grammes of potassium iodide to the litre), the temperature being normal and the pulse 74 . At 6 P.M. a second intrauterine douche was given, and three-quarters of an hour later tout était à recommencer-viz., temperature $102 \cdot 6^{\circ}$, violent rigor, and rapid pulse. I therefore decided once more to pack the uterus and thereby prevent the accumulation of fluid. This dressing - namely, an intra-uterine douche followed by packing with iodoform gauze-I repeated the next morning and for 48 hours had the pleasure of seeing my patient maintain a uniformly good condition with normal pulse and temperature, the milk having, however, completely left the breasts, showing the intensity of the general infection. On the following day I replaced the gauze packing by a long intra-uterine glass drain and a vaginal gauze plug, the pulse being 72. In deference to the opinion of my consultants, who strongly recommended intra-uterine douching and equally strongly deprecated drainage of the uterus as risking contamination from the vagina and from without, I now-on the eleventh day-removed all drainage and gave an intra-uterine douche of iodised solution. There was almost immediately a rise of temperature to $101^{\circ}$ and for 12 hours there was no discharge whatever. When the next douche was given the uterus was found once more to be filled with turbid, infective fluid. I therefore again inserted a glass drain and packed the vagina, and repeated this dressing on the following day. Discharge was now free and the temperature once more fell to normal. The patient's strength was, however, becoming exhausted and $I$ felt that if a repetition of the absurption from the uterus occurred great danger would be run. Fortunately I was given a free hand

1 The ecouvillon or mop is made of stout quill mounted on a wire stem. It thoroughly cleanses the uterine surface without the cutting effect of the steel instruments. It is readily sterilised by dry heat and is cheap, costing only $5 d$. They may be obtained at Messrs. Down Bros. or any Paris instrument maker. by both the patient and her family and I continued the drainage for six days, soon replacing the glass tube with diminishing amounts of iodoform gauze. The twice-daily douching and dressing were troublesome and trying to both my patient and to myself but we were rewarded by a persistently normal temperature and pulse, a gradually increasing strength and appetite, and a feeling of assurance that the danger was over.

The patient recovered with the same remarkable rapidity as that with which she fell ill and on the eighteenth day after ber confinement was able to be up. I may add that vaginal examination throughout detected no marked displacement of the uterus although the slight retroflexion present must have sufficed to kink the cervix and prevent the necessary discharge of lochia. Bacteriological examination of the fluid at the time of the curettage, on the eighth day, gave cultures of bacillus coli and micrococci of the tetrad group.

The interest of the case is great, for it is surely exceptional for the classical treatment of slight infection-namely, early curettage followed by intra-uterine douching-to fail. Not only did it fail but it seems morally certain, from the above record of the case, that it would have caused the patient's death or at any rate not prevented her death if persisted in.

In conclusion, if I may venture to point the moral, it is as follows: 1. Let the physician assure himself of the free flow of lochia in the days immediately following the confinement. Confidence in the assurance of the nurse upon this point may afterwards be regretted. 2. Drainage of the uterus, being simple, painless, and free from danger, should be practised early in certain cases.

Paris.

\section{A CASE OF CIRRHOSIS OF THE LIVER IN WHICH CURE OF THE ASCITES FOLLOWED AN OPERATION FOR THE RELIEF OF STRANGU- LATED UMBILICAL HERNIA.}

BY JOHN CLAY, M.B., B S. DURH., F.R C.S. ENG., ASSISTANT SURGEOY TO THE ROYAL VICTORIA INFIRMARY, NEWCASTLFON-TYNE; DEMONSTRATOR OF ANATOMY IN THE UNIVERSITY OF DURHAM.

ON Dec. 18th, 1906 I was asked by Dr. G. Armstrong Atkinson to see a patient who was suffering from strangulated umbilical hernia. The history of the patient, a man, aged 60 years, was that for four years he had suffered from ascites due to cirrhosis of the liver and during the latter part of the period the condition had become complicated by a hernia at the umbilicus. As so often happens in these cases, the present attack began very gradually with symptoms of obstruction of the lumen of the bowel, and it was only during the last day that signs of strangulation of the vessels supply. ing its walls appeared. On the night on which I saw the man he was very ill and as evidence of the strangulation a basin containing rather more than a pint of fæcal vomit was shown to me. There appeared to be little hope of a successful result for operation but none at all if affairs were allowed to take their course. The copious fæcal vomit absclutely contra-indicated a general anæsthetic, so I determined to relieve the condition, using a local anæsthetic only. $\beta$-eucaine was employed, about three grain boiled in one ounce of water with a little common salt. After cleaning the skin the neck of the sac was clearly exposed and the peritoneal cavity was opened immediately above it. From the peritoneal cavity the finger was passed into the sac which was then laid open together with its numerous ramifications, and the strangulation which was in one of them was relieved. The gut where strangulated was almost gangrenous, though recovery did not seem impossible. During the operation large quantities of ascitic flaid escaped. The bowel was returned to the abdominal cavity and the wound was sutured longitudinally in the usual way.

The patient, though somewhat wayward, made an excellent recovery and his medical attendant tells me to-day that he is better than he has been for some years and that no ascitic fluid has re-collected. The local anæsthetic acted well and no pain was felt in making the skin incision, bat the parietal peritoneum was extremely tender, considerably adding to the 
difficulty of in-erting the sutures. The bowels are not naturally sensitive and therefore no discomfort was felt from their handling.

The method of opening the peritoneal cavity on the proximal side of the hernial sac as an early step in the operation for the relief of strangulated hernia has often proved of service to me. In this case it shortened the duration of the operation, a matter of considerable importance when the anæsthetic is local and when the general condition of the patient is bad. The whole procedure only took 35 minutes.

In inguinal as well as in femoral hernia this method enables the bowel both above, below, and also at the site of the stricture to be inspected thoroughly, just as it lies, without pulling down of the constricted part and so running the risk of tearing the softened coats. In inguinal hernia there is no difficulty in opening the peritoneal cavity on the proximal side of the neck of the sac, but in femoral hernia the sac is so surrounded by important structures that at earlier operations I could not see any way of following out this principle in cases where it appeared necessary. However, in working at the anatomy of the part I saw that Poupart's ligament could easily be raised from the femoral vessels and space obtained towards the outer side of the femoral canal. The deep epigastric vessels may require division and the vas may need to be drawn aside. Enough room may be obtained in this way to get the hand into the abdominal cavity if necessary. In femoral hernia it is the narrowness of the neck of the sac which causes the constriction and not Gimbernat's ligament, which has so often been blamed. By incising the neck of the sac on the outer side, this ligament, which is as a fact an expansion of the inner attached end of Poupart's ligament, is spared and an almost perfect repair is made possible.

In a case of Richter's hernia I was able by this method of entering the peritoneal cavity to find the piece of bowel in which a small patch of the wall had sloughed, thus avoiding the necessity of dividing Poupart's ligament. This patient, whom Dr. R B. Lorraine and Dr. D. C. Welsh had asked me to see, made a good recovery - a rare event with this variety of hernia.

Newcastle-on-Tyne.

\section{Climital ghotes: \\ MEDICAL, SURGICAL, OBSTETRICAL, AND THERAPEUTICAL}

A CASE OF MALFORMATION OF THE THUMB. By BRYAN ProK, M.R.O.S. ENG., L.R.C.P. LOND., SURGEON; R.N.

THE following malformation came under notice quite recently in a case in which a very curious history was given. The man states that before he was born his mother was bitten by a crab. The left thumb is not unlike a crab's claw. There

FIG. 1.

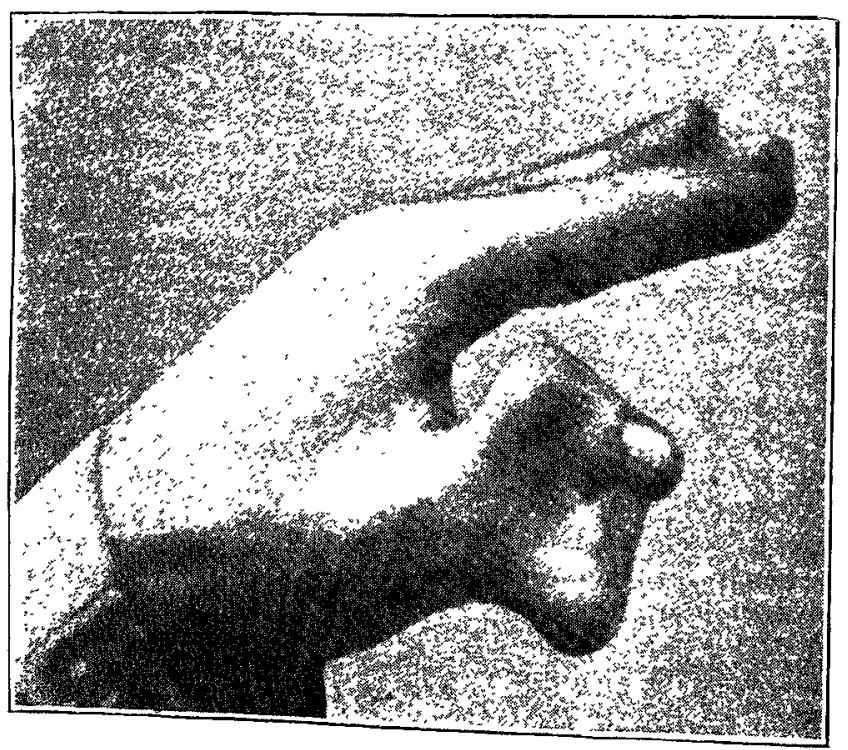

are two distinct ungual phalangts, ench bearing a nail. There are two joints which allow of u certain amount of movement he being able to pick up small articles with the two "thumbs." The first phalanx is double as far as the base where it articulates with the metacarpal bone in the ordinary way. The interspace is filled in by a web. (Fig. 1.) It seems that the two shafts of the phalanx have been developed from separate centres and that the base has only had one centre,

FIG. 2.

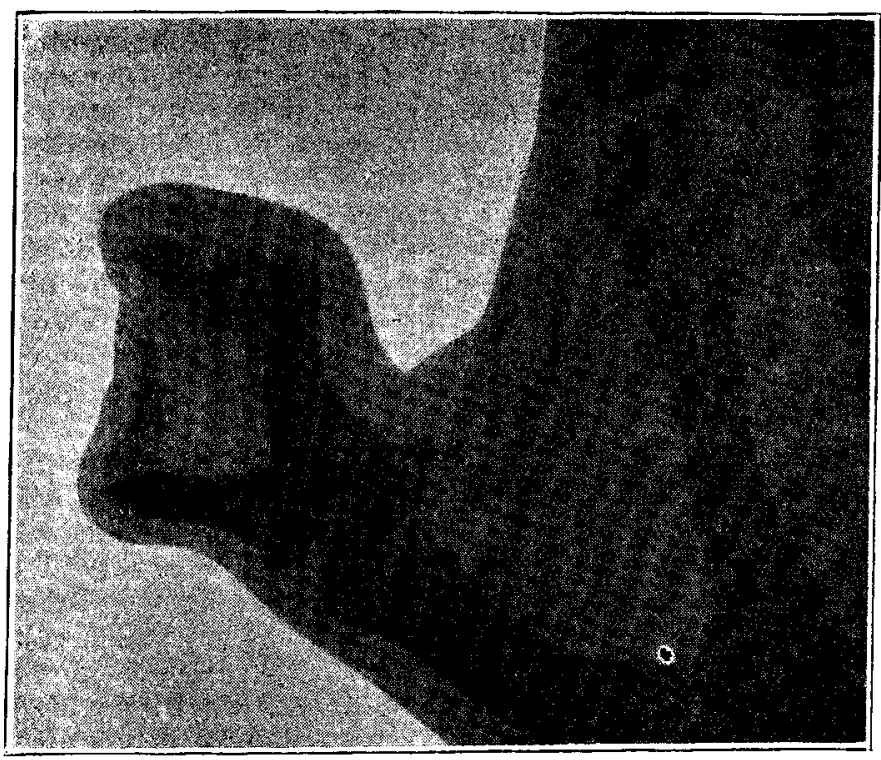

as there does not appear to be any sign showing two centres which have subsequently become fused. (Fig. 2.) The condition causes the man no inconvenience and he has no other abnormality. There are no means of finding out at what period of the mother's pregnancy the incident took place.

HæMOPHILIA TRANSMITTED THROUGH THE MALE.

\section{By A. J. Swanton, L.R.C.P.\& S. IREL.}

THE following note of a hæmophilic family that has recently come under my notice presents an example of an unusual method of transmission in this disease. It arose de novo in $\mathrm{S} . \mathrm{P}$., in whose parentage or ancestry no history of it could be traced; he suffered from severe hæmorrhage from the most trivial cut and also from epistaxis. He had 13 children, ail of whom were free from the affection with the exception of one son, J. P., who was a "bleeder" from infancy; it is a pious opinion in his family that this fact was due to the father, who was suffering from a brisk epistaxis, having rushed home and frightened his wife who was then pregnant of the son, J. P. I had no personal knowledge of S. P.'s case, but know the son, who is now over 40 years of age, and his family, which consists of six girls and one boy, varying in age from two months to 19 years, all of whom show symptoms of bæmophilia except one girl aged eight years. Four have died, two boys at eight months and four years respectively, from "convulsions." said to have been due to epistaxis, and one boy, aged ten months, and a girl, aged two years and eight months, from pneumonia following measles accompanied by severe epistaxis and bleeding from the mouth.

The disease in J. P. chiefly takes the form of spontaneous epistaxis which is difficult to control and is preceded by malaise and headache. He also suffers from painful swelling of his large joints. All the children look healthy; they have very soft white skin and fair hair. The only surviving son suffers frequently from epistaxis, which is most alarming in its persistence. It is preceded, for a few days, by an elevated temperature with frontal headache, faintness, loss of appetite, and pain in the back. The elbows, wrists, knees, and ankles are swollen and very painful. With the onset of bleeding he perspires freely. Any form of traumatic bleeding is only controlled with the greatest difficulty. $\mathrm{He}$ also bleeds per anum when suffering from diarrhœi. A baby (girl), aged two months, has been subject to attacks of epistaxis from birth. The two girls, aged five years and 14 years respectively, suffer from troublesom? attraks of bleeding, the chief form being epistaxis, and from the U 4 